\title{
A $\sigma$-Aromatic Tri-Thorium Bonding: Does It Really Exist?
}

\author{
Dariusz W. Szczepanik ${ }^{1,2^{*}}$
}

1. K. Guminski Department of Theoretical Chemistry, Faculty of Chemistry, Jagiellonian University, Gronostajowa, 2, 30-387 Kraków, Poland.

2. Institut de Quìmica Computacional i Catàlisi and Departament de Química, Universitat de Girona, C/ Maria Aurèlia Capmany, 69, 17003 Girona, Catalonia, Spain.

Corresponding author's email: dariusz.szczepanik@uj.edu.pl 
The experimental realization of actinide-actinide bonding in isolable molecules has been one of the main targets of synthetic actinide chemistry for decades ${ }^{1,2}$. Very recently, Boronski et al. reported the first thorium-thorium bonding in a crystalline cluster prepared and isolated under normal experimental conditions, $\left[\left\{\mathrm{Th}\left(\eta^{8}-\mathrm{C}_{8} \mathrm{H}_{8}\right)\left(\mu_{3}-\mathrm{Cl}\right)_{2}\right\}_{3}\left\{\mathrm{~K}(\mathrm{THF})_{2}\right\}_{2}\right]_{\infty}(3)^{3}$. The electron spin resonance and the superconducting quantum-interference device magnetometry revealed a diamagnetic ground-state with two electrons equally distributed over the tri-thorium ring at the heart of $\mathbf{3}$, and the recorded Raman spectrum allegedly confirmed the existence of a three-center two-electron (3c-2e) bond. However, the broad inelastic scattering bands centered at 72 and 105 $\mathrm{cm}^{-1}$, originally assigned by the authors to Th-Th vibrations, represent in fact the combination of Th- $\mathrm{Cl}$ stretching and $\mathrm{Cl}-\mathrm{Th}-\mathrm{Cl}$ bending, and as demonstrated in the following, they establish the existence of a unique charge-shift actinide-halogen bonding rather than the $\sigma$-aromatic (delocalized) actinide-actinide bonding as claimed in the original article ${ }^{3}$.

To understand the electronic structure of $\mathbf{3}$, Boronski et al. computationally investigated several model clusters, including $\left[\left\{\mathrm{Th}\left(\eta^{8}-\mathrm{C}_{8} \mathrm{H}_{8}\right)(\mu-\mathrm{Cl})_{2}\right\}_{3} \mathrm{~K}_{2}\right]\left(3^{\prime \prime}\right)$ with the Highest Occupied Molecular Orbital (HOMO) reminiscent of a 3c-2e bond, and its dication $\left[\left\{\mathrm{Th}\left(\eta^{8}-\mathrm{C}_{8} \mathrm{H}_{8}\right)(\mu-\right.\right.$ $\left.\left.\mathrm{Cl})_{2}\right\}_{3} \mathrm{~K}_{2}\right]^{2+}$ (here denoted by $3^{*}$ ) without the characteristic $3 \mathrm{c}-2 \mathrm{e}$ HOMO. The authors found that the structure of 3" better fits the X-ray diffraction data and the calculated vibration modes match well with the intensive signals observed in the experimental Raman spectrum, which they interpreted as an "experimental confirmation of the Th, bonding in 3". However, such an interpretation is mostly inaccurate as the same vibration modes feature the model cluster $3^{*}$, which lacks any direct actinide-actinide bonding (the reported thorium-thorium bond orders for 3" and $\mathbf{3}^{*}$ are 0.254 and 0.039, respectively). As shown in Figure 1a (and in the Supplementary Video clip), the symmetric breathing modes centered at 77 and $107 \mathrm{~cm}^{-1}$ in $\mathbf{3}$ " can easily be 
found respectively at 75 and $103 \mathrm{~cm}^{-1}$ in $\mathbf{3}^{*}$, although the lack of delocalized and highly polarizable HOMO in the latter makes them hardly detectable in Raman spectroscopy. Furthermore, the authors overlooked the fact that the displacement vectors on thorium and chlorine atoms in both model clusters have comparable magnitudes, which strongly suggests that the observed broad inelastic scattering bands between 60 and $135 \mathrm{~cm}^{-1}$ represent collective stretching and bending modes of the actinide-halogen rather than actinide-actinide bonding. This becomes particularly evident if one compares the overall bond order in the tri-thorium ring in $\mathbf{3}$ ", $3 \times 0.254=0.762$, with twelve polarized thorium-chlorine bonds, $12 \times 0.599=7.188$ (here the full bond order of 0.599 is the sum of the reported by the authors covalent bond order of 0.374 and the ionic component estimated from the modified Evarestov-Veryazov equation ${ }^{4}, 0.225$ ). These numbers clearly show that more than $90 \%$ of bonding interactions in the entire $\mathrm{Th}_{3} \mathrm{Cl}_{6}$ cage in the model cluster 3 " are associated with the thorium-chlorine bonds (for $3 *$ it is $99 \%$ ), which actually explains the very small frequency shifts between the corresponding symmetric vibration modes in $\mathbf{3}^{\text {" and }} \mathbf{3}^{*}$. It should also be noticed that the overall vibrational contributions to thermal energy in $3^{\prime \prime}\left(286.5 \mathrm{kcal} \mathrm{mol}^{-1}\right)$ and $\mathbf{3}^{*}\left(287.3 \mathrm{kcal} \mathrm{mol}^{-1}\right)$ differ by less than $1 \mathrm{kcal} \mathrm{mol}^{-}$ ${ }^{1}$, which further confirms extremely weak tri-thorium bonding (if any) and its marginal effect on the thermodynamic stability of $\mathbf{3}$. 

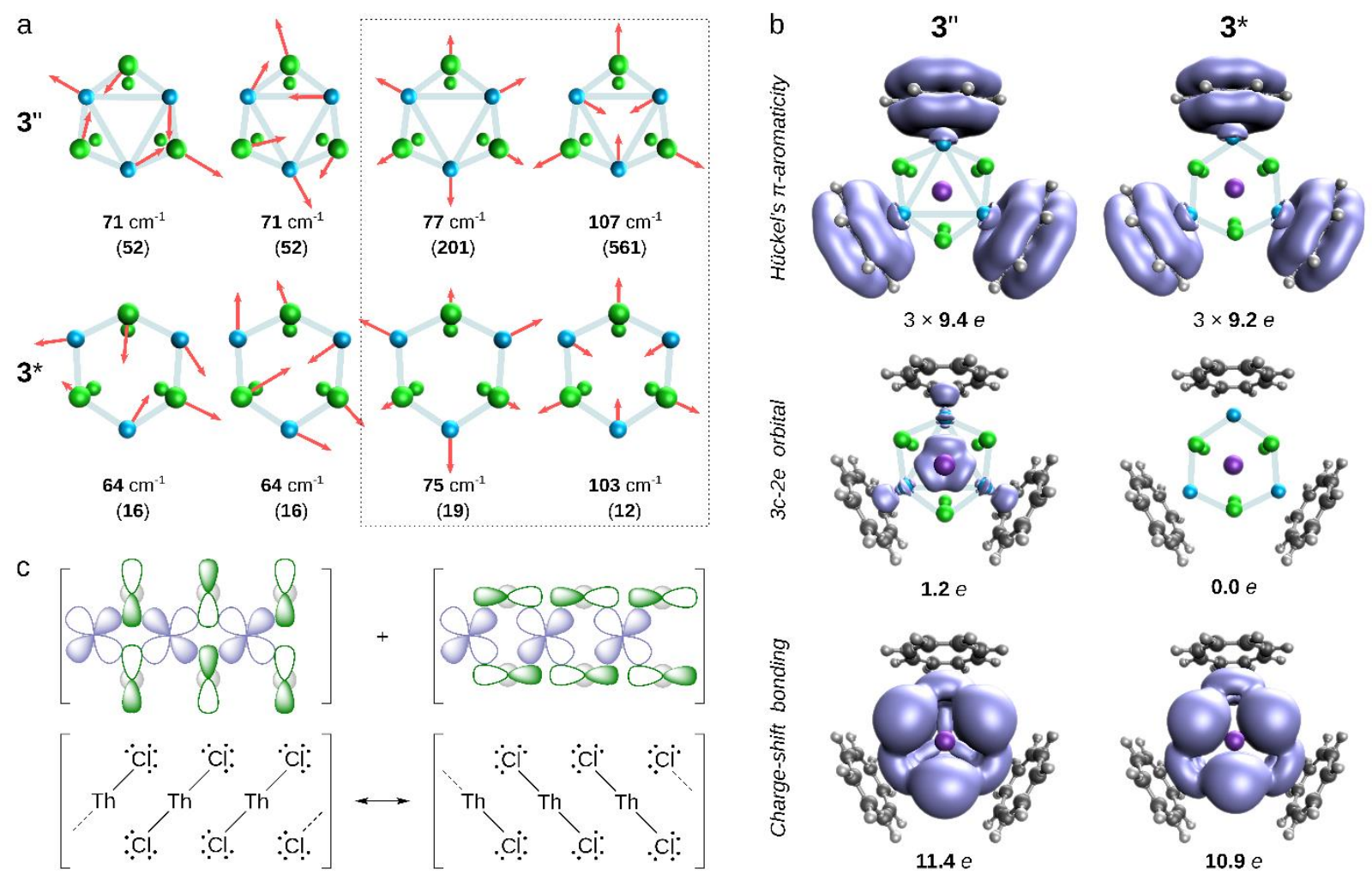

Figure 1. (a) Selected molecular vibration modes in 3 " and $3^{*}$ with the corresponding Raman scattering activities in brackets (in units $\mathrm{A}^{4} \mathrm{AMU}^{-1}$ ). (b) Isosurfaces (at $0.003 e$ ) of the EDDB function in $3^{\text {" }}$ and $3^{*}$ dissected into separate contributions to global electron delocalization from the $\mathrm{C}_{8} \mathrm{H}_{8}{ }^{2-}$ ligands, $\mathrm{Th}_{3}$ ring, and the entire $\mathrm{Th}_{3} \mathrm{Cl}_{6} \mathrm{Cage}_{\text {(with the }}$ $\mathrm{Th}_{3}$ delocalization excluded). (c) The linearized side-view of two orbital conjugation topologies in which the overlap of the $6 \mathrm{~d}(\mathrm{Th})$ and $3 \mathrm{p}(\mathrm{Cl})$ atomic orbitals is expected to be particularly effective; below the corresponding resonance Lewis structures that rationalize the delocalized ("resonating") nature of the thorium-chlorine bonding in the $\mathrm{Th}_{3} \mathrm{Cl}_{6}$ cage in $\mathbf{3}^{\prime \prime}$ and $\mathbf{3}^{*}$.

In the light of the above clarification, the existence of the tri-thorium bonding in the synthetized crystalline actinide cluster remains experimentally unproven. Going further, the uniform charge distribution over the tri-thorium ring in $\mathbf{3}$ " is evidently forced by highly symmetric $\left(\mathrm{D}_{3 \mathrm{~h}}\right)$ arrangement of the thorium-chlorine bonds in the $\mathrm{Th}_{3} \mathrm{Cl}_{6}$ cage, and as such it may have nothing to do with the aromatic stabilization effect. In this context, negative values of the nucleus-independent chemical shift $(\mathrm{NICS})^{5}$ reported by the authors are meaningless as NICS is unable to distinguish between the magnetic shielding caused by the aromatic ring current and 
the local circulations around the lone-pairs on the surrounding chlorine atoms ${ }^{6}$. This is a wellknown issue of NICS, and for this reason the quantities based on electron density are much more reliable probes of aromaticity in the metal clusters ${ }^{7}$. For instance, the electron density of delocalized bonds $(\mathrm{EDDB})^{8}$, presented in Figure 1b, provides very clear and distinct pictures of electron delocalization in 3 " and $\mathbf{3}^{*}$ that fully confirm the dominating role of the delocalized thorium-chlorine bonding in the $\mathrm{Th}_{3} \mathrm{Cl}_{6}$ cage, which is comparable in magnitude to the cyclic delocalization in the $\pi$-aromatic $\mathrm{C}_{8} \mathrm{H}_{8}{ }^{2-}$ ligands, and an order of magnitude higher than in $\mathrm{Th}_{3}$.

As shown in Figure 1c, the delocalized nature of the $\mathrm{Th}_{3} \mathrm{Cl}_{6}$ bonding can easily be rationalized on the ground of the chemical resonance theory: the polarized bonds $\mathrm{Th}-\mathrm{Cl}$ and the corresponding lone-pairs on chlorine atoms cooperatively switch their positions giving rise to the formal half-bonding between thorium and chlorine atoms, which is fully consistent with the estimated above full bond order of $\mathrm{Th}-\mathrm{Cl}(0.599)$. This type of bonding is known in the literature as a charge-shift bond, ${ }^{9,10}$ and it has recently been shown to be particularly strong when $5 \mathrm{~d}$ orbitals of the transition-metals from groups 11 and 12 are involved. ${ }^{11}$ Therefore, the unwittingly discovered by Boronsky et al. delocalized actinide-halogen bonding pattern extends the range of the charge-shift bonding beyond transition metals to the seventh row of the periodic table.

To summarize, by clarifying inaccurate and misleading conclusions of the original Nature paper $^{3}$ and drawing special attention to the essential role of actinide-halogen charge-shift bonding, this study may have broader implications for understanding the chemistry of actinides and future attempts to design and synthesize new stable actinide complexes.

\section{Data Availability}

All the data investigated within this paper come from the original paper by Boronsky et al. ${ }^{3}$ or were calculated using the same methodology, computational level and the quantum-chemical 
software. The supplementary video clip visualizes the symmetric vibration modes at 77 and 107 $\mathrm{cm}^{-1}$ in 3" (left column), and the corresponding modes at 75 and $103 \mathrm{~cm}^{-1}$ in $3^{*}$ (right column). The results of the bond order and electron delocalization analyses were validated in the fully relativistic Douglas-Kroll-Hess 4th-order calculations (including the spin-orbit coupling terms and the fully relativistic basis set of the triple-zeta quality), and the resulting data are available from the corresponding author upon reasonable request.

\section{Acknowledgments}

The author expresses his special thanks to Prof Stephen Liddle, Prof. Miquel Solà, Dr. Josef T. Boronski, and Dr. Marcin Andrzejak for stimulating discussions and critical reading of the first draft of the manuscript. The research was supported by the Polish National Agency for Academic Exchange under the Bekker programme, Grant Agreement No.

PPN/BEK/2019/1/00219 and the PL-Grid Infrastructure of the Academic Computer Centre (CYFRONET).

\section{References}

1. Cavigliasso, G., Kaltsoyannis, N. On the paucity of molecular actinide complexes with unsupported metal-metal bonds: a comparative investigation of the electronic structure and metal-metal bonding in $\mathrm{U}_{2} \mathrm{X}_{6}\left(\mathrm{X}=\mathrm{Cl}, \mathrm{F}, \mathrm{OH}, \mathrm{NH}_{2}, \mathrm{CH}_{3}\right)$ complexes and d-block analogues. Inorg. Chem. 45, 6828-6839 (2006).

2. Huh, D. N., Roy, S., Ziller, J. W., Furche, F. \& Evans, W. J. Isolation of a square-planar $\mathrm{Th}(\mathrm{III})$ complex: synthesis and structure of $\left[\mathrm{Th}\left(\mathrm{OC}_{6} \mathrm{H}_{2}{ }^{\mathrm{t}} \mathrm{Bu}_{2}-2,6-\mathrm{Me}-4\right)_{4}\right]^{1-}$. J. Am. Chem. Soc.

141, 12458-12463 (2019). 
3. Boronski, J.T., Seed, J.A., Hunger, D., Woodward A.W., van Slageren J., Wooles A.J., L.S. Natrajan, Kaltsoyannis N., Liddle S.T. A crystalline tri-thorium cluster with $\sigma$-aromatic metalmetal bonding. Nature 598, 72-75 (2021).

4. Szczepanik, D.W., Mrozek, J. Probing the interplay between multiplicity and ionicity of the chemical bond. J. Theor. Comput. Chem. 10, 471-482 (2011).

5. Chen, Z., Wannere, C. S., Corminboeuf, C., Puchta, R., Schleyer, P. von R. NucleusIndependent Chemical Shifts (NICS) as an Aromaticity Criterion. Chem. Rev. 105, 3842-3888 (2005).

6. Cuyacot, A.B.J., Foroutan-Nejad, C. Aromatic, or Antiaromatic, That Is the Question. Preprint at http://doi.org/10.33774/chemrxiv-2021-3gv6v (2021).

7. Feixas, F., Jiménez-Halla, J.O.C., Matito, E., Poater, J., Solà, M. A test to evaluate the performance of aromaticity descriptors in all-metal and semimetal clusters. An appraisal of electronic and magnetic indicators of aromaticity. J. Chem. Theory Comput. 6, 1118-1130 (2010).

8. Szczepanik, D.W., Andrzejak, M., Dyduch, K., Zak, E.J., Makowski, M., Mazur, G., Mrozek, J. A uniform approach to the description of multicenter bonding. Phys. Chem. Chem. Phys. 16, 20514-20523 (2014).

9. Shaik, S., Danovich, D., Wu, W., Hiberty, P.C. Charge-shift bonding and its manifestations in chemistry. Nature Chem. 1, 443-449 (2009).

10. Shaik, S., Danovich, D., Galbraith, J.M., Braïda, B., Wu, W., Hiberty, P.C. Charge-shift bonding: a new and unique form of bonding. Angew. Chem. Int. Ed. 59, 984-1001 (2020). 
11. Joy, J., Danovich, D., Kaupp, M., Shaik, S., Covalent vs Charge-Shift Nature of the MetalMetal Bond in Transition Metal Complexes: A Unified Understanding. J. Am. Chem. Soc. 142, 12277-12287 (2020). 